\title{
EVIDENCE FOR MAGNETIC FIELDS IN THE OUTFLOW FROM T TAU S
}

\author{
T.P. RAY
}

Dublin Institute for Advanced Studies

T.W.B. MUXLOW

National Radio Astronomy Laboratory, Jodrell Bank

D.J. AXON

Space Sciences Division of ESA,

Space Telescope Science Institute, Baltimore

\section{A. BROWN}

Center for Astrophysics and Space Astronomy,

University of Colorado

D. CORCORAN

Dublin Institute for Advanced Studies

J. E. DYSON

Department of Physics and Astronomy, University of Leeds

AND

R. MUNDT

Max-Planck-Institut für Astronomie, Heidelberg

\begin{abstract}
We have observed at $5 \mathrm{GHz}$ the $\mathrm{T}$ Tau system with high resolution ( $\left.\lesssim 0^{\prime \prime} .1\right)$ using the Multi-Element Radio Linked Interferometer (MERLIN) based at Jodrell Bank. Both the optical star (T Tau N) and its wellknown infrared companion (T Tau $\mathrm{S}$ ) were detected. The radio emission from T Tau S was found to be roughly extended in the direction of what is thought to be its outflow axis. More importantly we discovered that this radio emission split up into two spatially separated lobes of opposite helicity in the left and right-circular polarization channels. The circularly polarized lobes appear to straddle the star so that the "flow" and the "counterflow" were of opposite helicity. Such observations are the first direct evidence for the presence of magnetic fields in extended outflows that we are aware of. The radio flux appears to be due to gyrosynchrotron emission from mildly
\end{abstract}


relativistic electrons $(\gamma \approx 2-3)$. These electrons may have been accelerated in shocks close to the source. Using reasonable assumptions, the inferred magnetic fields strengths are surprisingly large ( $\gtrsim$ several gauss) at distances of approximately 10-20 AU from their source. This is consistent with the magnetic fields being part of a collimated flow.

\section{Introduction and Observational Details}

$\mathrm{T}$ Tau is in many ways not typical of the class of stars to which it gives it name and it is perhaps because of its enigmatic nature that it remains an object of intense study. It is associated not only with a well-known reflection nebula (Hind's Nebula or NGC 1555) but it is at the centre of a diffuse HH region (Burnham's Nebula) powered by shocks (Solf et al., 1988; Böhm \& Solf, 1994) and a complex system of interlocking loops is seen in the near-infrared (see the poster by Herbst et al., 1997). T Tau has been shown using infrared speckle interferometry to have a companion which lies approximately $0^{\prime \prime} .7$ south (Ghez et al., 1991) of the visible star. Proper motion studies (Ghez et al., 1995) have implied that the optical star and T Tau S almost certainly form a gravitationally bound system, the projected separation of which is decreasing at a rate of approximately $8 \mathrm{mas} / \mathrm{yr}$. Both the optical star and its infrared companion have been detected at radio wavelengths (Schwartz et al., 1984; Skinner \& Brown, 1994) and these radio sources are referred to as $\mathrm{T}$ Tau $\mathrm{N}$ and $\mathrm{T}$ Tau $\mathrm{S}$ respectively. The centimeter emission from $\mathrm{T}$ Tau $\mathrm{S}$ is known to be variable on timescales of a few days and to be circularly polarized (Philips et al., 1993; Skinner \& Brown, 1994).

In November 1992, we used the Multi-Element Radio Interferometer Network (MERLIN) based at Jodrell Bank to map T Tau at $6 \mathrm{~cm}$ in phase referencing mode. Details are contained in Ray et al. (1997). Our final smoothed beam size was $0^{\prime \prime} .2$ and the $\mathrm{rms}$ noise level was $170 \mu \mathrm{Jy} /$ beam. The total field of view was $7^{\prime \prime} .68 \times 7^{\prime \prime} .68$.

\section{Results}

Our $6 \mathrm{~cm}$ radio map of the $\mathrm{T}$ Tau region is shown in Figure l. The two previously known radio sources $\mathrm{T}$ Tau $\mathrm{N}$ and $\mathrm{T}$ Tau $\mathrm{S}$ can be seen, along with extended emission and a third source which we have labeled T Tau R. It is not clear whether $\mathrm{T}$ Tau $\mathrm{R}$ is another star in the $\mathrm{T}$ Tau system or perhaps part of an outflow. If it is a highly embedded star (note that it was not seen using near-infrared speckle interferometry) then it may have been confused in the past with T Tau N (Ray et al., 1997). The positions 


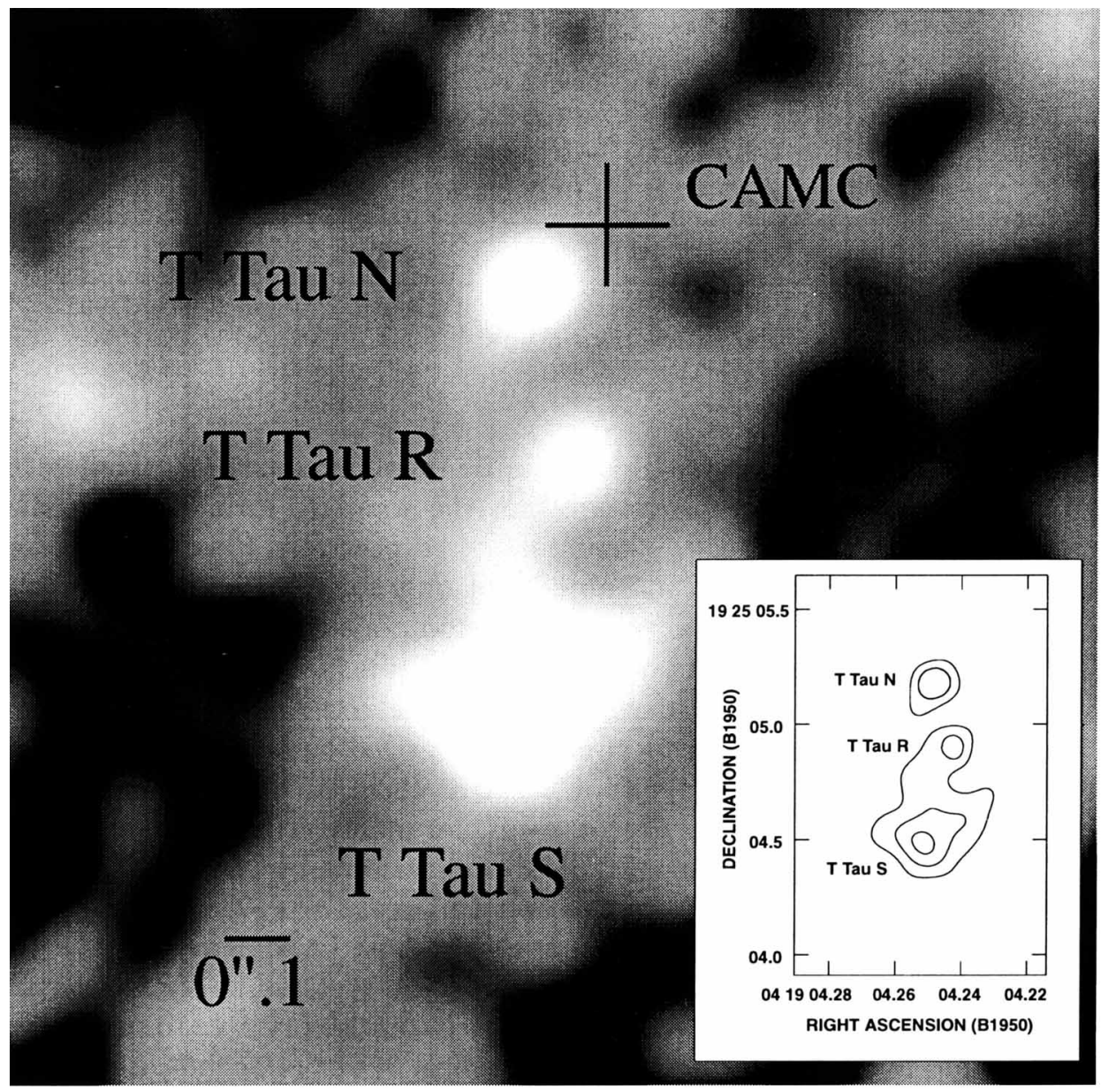

Figure 1. MERLIN $6 \mathrm{~cm}$ intensity image of T Tau from Ray et al. (1997). Apart from the known radio sources $\mathrm{T}$ Tau $\mathrm{N}$ and $\mathrm{T}$ Tau $\mathrm{S}$, extended radio emission is observed between these two stars. There is also a third source which may have been wrongly classified as the optical star in the original VLA discovery image (see text and Ray et al., 1997). The radio emission from $T$ Tau $S$ appears extended in the northwest-southeast direction due to its outflow (see also Bally \& Devine, this volume). The position of the optical star, as derived from the Carlsberg Automatic Meridian Circle, and allowing for its known proper motion, is also shown. The $1 \sigma$ errors in the optical position are indicated by the cross. Inset is a contour plot of the same data of the $\mathrm{T}$ Tau region; contours are at 0.4 , 0.7 and 1.2 milliJanskys.

of the various radio sources, along with that of the optical star, are listed in Table l. The optical position of T Tau, allowing for its proper motion, is derived from Carlsberg Automatic Meridian Circle data (Ray et al., 1997) and it is also marked in Figure 1. From the overlap in their positions, it is 


\begin{tabular}{|c|c|c|c|c|c|}
\hline Source & $\mathrm{RA}(\mathrm{B} 1950)$ & $\operatorname{Dec}(\mathrm{B} 1950)$ & $\mathrm{I}(\mathrm{mJy})$ & $\mathrm{R}(\mathrm{mJy})$ & $\mathrm{L}(\mathrm{mJy})$ \\
\hline T Tau N & 041904.246 & +192505.17 & 0.9 & 0.5 & 1.3 \\
T Tau R & 041904.243 & +192504.88 & 0.8 & 0.6 & 1.1 \\
T Tau S & 041904.246 & +192504.54 & 2.1 & 2.4 & 1.8 \\
T Tau (Optical) & 041904.240 & +192505.26 & - & - & - \\
\hline
\end{tabular}

TABLE 1. MERLIN determined positions and $6 \mathrm{~cm}$ flux densities for sources in the T Tau region. All positions quoted are Equinox B1950.0 and Epoch J1992.9 i.e. the epoch of the radio observations

clear that the radio source $\mathrm{T}$ Tau $\mathrm{N}$ can be unambiguously identified with the optical star. We should mention that the standard errors in the radio and optical positions are $\pm 0 .{ }^{\prime \prime} 035$ and $\pm 0^{\prime \prime} .1$ respectively. Flux densities for the 3 sources are also given in Table 1 not only for total intensity (I) but right $(\mathrm{R})$ and left $(\mathrm{L})$ circular polarization components as well (see below). The typical errors in the flux densities are $\pm 0.15 \mathrm{mJy}$.

We have already referred to the fact that the radio emission from $\mathrm{T}$ Tau S has been known to be circularly polarized on occasions. VLA observations by Philips et al. (1993) of T Tau at $18 \mathrm{~cm}$, although not resolving the emission, showed that its degree of circular polarization to be very high. Interestingly nearly simultaneous VLBI observations by the same authors overresolved the emission. From this fact Philips et al. (1993) argued that the size of the $\mathrm{T}$ Tau $\mathrm{S}$ radio emitting region must lie somewhere between the resolutions afforded by the VLBI and VLA systems i.e. from tens of stellar radii to a few tens of AU. Somewhat to our surprise, examination of the left and right circularly polarized channels of our radio image, showed that the emission from $\mathrm{T}$ Tau $\mathrm{S}$ divided up into two spatially separated lobes of opposite helicity (see Figure 2). The emission centroids of the two lobes were approximately $0 " .15$ apart corresponding to a distance of just over 20AU if we assume the Taurus-Auriga Cloud is 140pc away.

As far as we are aware, this is the first time that extended i.e. resolved polarization has been observed at radio wavelengths in the vicinity of a young stellar object (YSO). Moreover it clearly implies the presence of magnetic fields on scales of tens of AU rather than a few stellar radii. As pointed out by Skinner \& Brown (1994) the only feasible mechanism for generating the observed emission is gyrosynchrotron radiation from electrons with mildly relativistic Lorentz factors i.e. $\gamma \approx 2-3$. As shown by Ray et al. (1997), the inferred magnetic field strengths are then of order several gauss at 10-20AU. Such fields are likely to be dynamically important and are certainly consistent with magnetic collimation models (see, for example, Ouyed et al., 1997 and Pudritz \& Ouyed, this volume) in which 


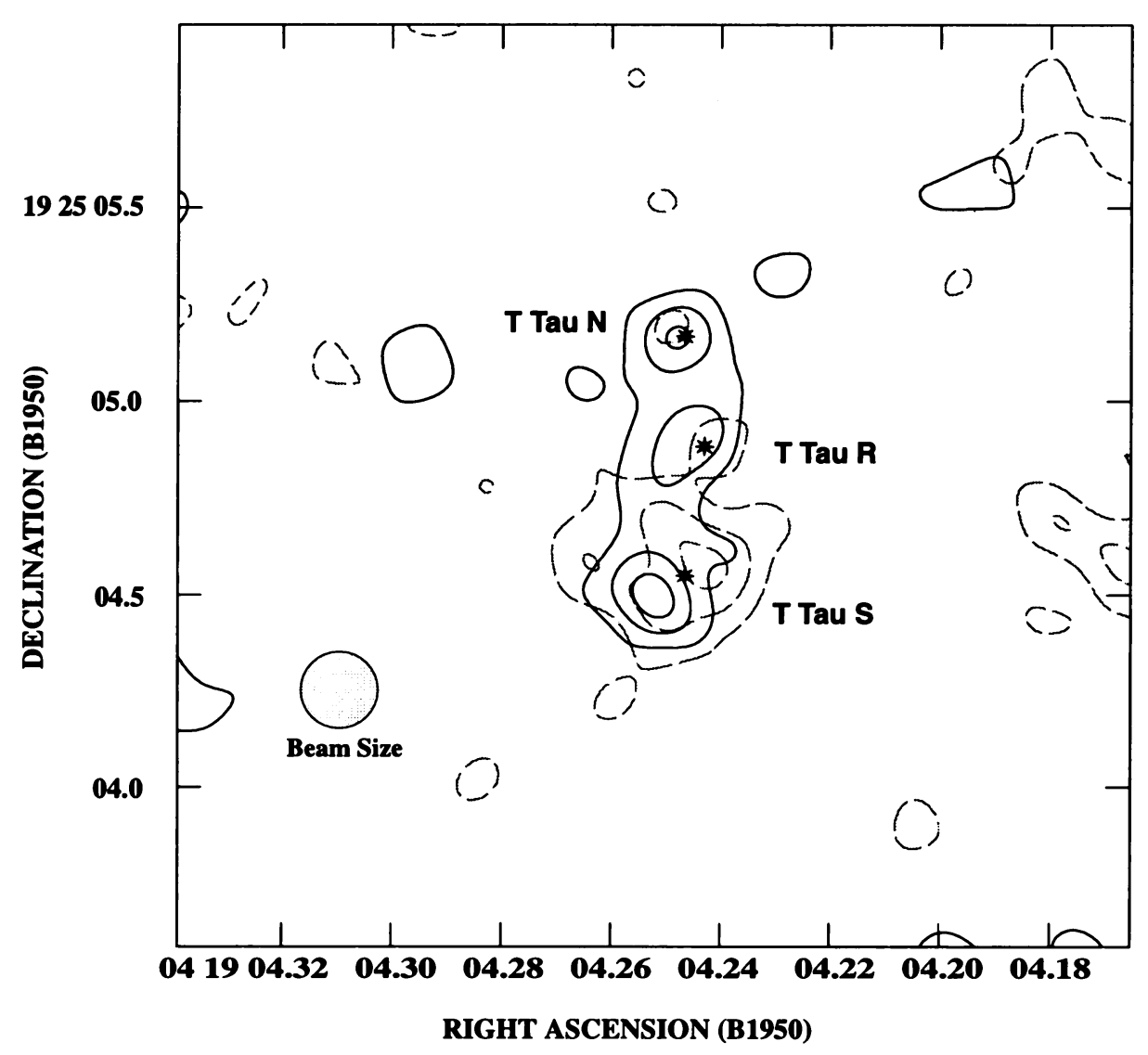

Figure 2. Our MERLIN contour map of the $6 \mathrm{~cm}$ radio emission in the T Tau region. Right and left circularly polarized components are shown as dashed and continuous lines respectively. Contour levels are in each case $0.4,0.8$ and $1.2 \mathrm{mJy}$. Note the spatially resolved separation of the emission from $\mathrm{T}$ Tau $\mathrm{S}$ into two lobes of opposite helicity and the net left-hand circular polarization of $T$ Tau $N$. Thus it appears that the 'flow' and 'counterflow' from T Tau S are both circularly polarized but in opposite senses. The source positions are shown as asterisks. Adapted from Ray et al. (1997).

outflows are produced, through the generation of a wind, by the winding up of magnetic fields anchored in an accretion disk. It is not clear at this stage whether the mildly relativistic electrons we observed in the outflow from $\mathrm{T}$ Tau $\mathrm{S}$, were accelerated in reconnection events close to the star (i.e. at distances of at most a few $R_{\star}$ ) or whether they received their energy in the outflow through diffusive shock acceleration.

The strength of the inferred fields at distances of 10-20 AU clearly points to them being part of a collimated flow (Ray et al., 1997) although we also 
emphasize that the fields may have been to some degree amplified through radiative shocks. It is also worth pointing out that $\mathrm{T}$ Tau $\mathrm{S}$ underwent what was probably an EX Lupi type outburst in 1990/91 due, presumably, to an increase in its accretion rate. In this regard it is interesting that while $\mathrm{T}$ Tau S appeared point-like in radio maps taken just before and around the time of the 1990 outburst (Skinner \& Brown, 1994), it is extended along the outflow direction in our radio image two years later. Thus the radio "lobes" may have had their origin in the EX Lupi type outburst.

Finally, we remark that a small number of other YSO outflows are known to have non-thermal radio spectra (see, for example, Curiel et al., 1993). Whereas linear polarization has been looked for but not found in these flows (e.g., the poster paper by Wilner et al., 1997) it could be the case that some outflows are emitting gyrosynchrotron rather than synchrotron radiation. If this is so then we should be looking for circular as opposed to linear polarization.

\section{References}

Böhm, K. H. and Solf, J., 1994, ApJ 430, 277

Curiel, S., Rodríguez, L. F., Moran, J. M. and Canto, J., 1993, ApJ 415, 191

Dyck, H. M., Simon, T. and Zuckerman, B., 1982 ApJ 255, L103

Ghez, A. M., Neugebauer, G., Gorham, P. W., Haniff, C. A., Kulkarni, S. R., Matthews, K., Koresko, C. and Beckwith, S., 1991, AJ 102, 2066

Herbst, T. M., Robberto, M. and Beckwith, S. V. W., 1997, Low Mass Star Formation from Infall to Outflow, Poster Proceedings of IAU Symp. No. 182, eds. F. Malbet \& A. Castets, p215

Ghez, A. M., Weinberger, A. J., Neugebauer, G., Matthews, K. and McCarthy, D. W., JR., 1995, AJ 110, 753

Königl, A. and Ruden, S. P., 1993, Protostars and Planets III, eds. Levy, E. \& Lunine, J., University of Arizona Press, 641

Ouyed, R., Pudritz, R. E. and Stone, J. M., 1997, Nature, 385, 409

Philips, R. B., Lonsdale, C. J. and Feigelson, E. D., 1993, ApJ 403, L43

Ray, T. P., Muxlow, T. W. B., Axon, D. J., Brown, A., Corcoran, D., Dyson, J. and Mundt, R., 1997, Nature, 385, 415

Schwartz, P. R., Simon, T., Zuckerman, B. and Howell, R.R., 1984, ApJ 280, L23

Skinner, S.L. and Brown, A., 1994, AJ, 107, 1461

Solf, J., Böhm, K. H. and Raga, A. C., 1988, ApJ 334, 229

Wilner, D.J., Reid, M.J., Menten, K.M. and Moran, J.M., 1997, Low Mass Star Formation - from Infall to Outflow, Poster Proceedings of IAU Symp. No. 182, eds. F. Malbet \& A. Castets, p193 